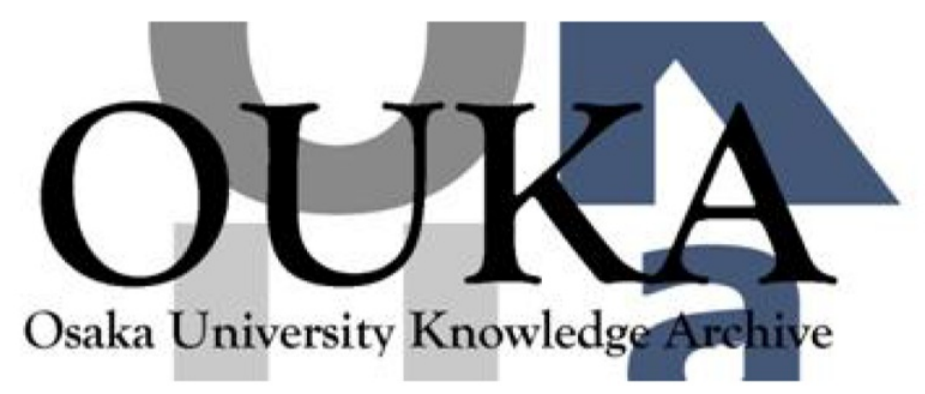

\begin{tabular}{|c|l|}
\hline Title & Squeezed spin states \\
\hline Author(s) & Kitagawa, Masahiro; Ueda, Masahito \\
\hline Citation & Physical Review A. 47(6) p.5138-p. 5143 \\
\hline Issue Date & $1993-06-01$ \\
\hline oaire:version & VoR \\
\hline URL & https://hdl. handle. net/11094/77656 \\
\hline rights & $\begin{array}{l}\text { Copyright (1993) by the American Physical } \\
\text { Society }\end{array}$ \\
\hline Note & \\
\hline
\end{tabular}

Osaka University Knowledge Archive : OUKA

https://ir. Library. osaka-u. ac. jp/

Osaka University 


\title{
Squeezed spin states
}

\author{
Masahiro Kitagawa and Masahito Ueda \\ Nippon Telegraph and Telephone Corporation Basic Research Laboratories, Musashino, Tokyo 180, Japan
}

(Received 12 February 1991; revised manuscript received 3 December 1992)

\begin{abstract}
The basic concept of squeezed spin states is established and the principles for their generation are discussed. Two proposed mechanisms, referred to as one-axis twisting and two-axis countertwisting, are shown to reduce the standard quantum noise $S / 2$ of the coherent $S$-spin state down to $\frac{1}{2}(S / 3)^{1 / 3}$ and $\frac{1}{2}$, respectively. Implementations of spin squeezing in interferometers are also discussed.

42.50.Dv, 03.65.Bz
\end{abstract}

\section{INTRODUCTION}

Squeezing, which redistributes quantum fluctuations between two noncommuting observables while preserving the minimum uncertainty product, has been extensively studied in boson systems [1]. A radiation field is said to be squeezed if the uncertainty of one quadrature amplitude $\left\langle\Delta a_{i}{ }^{2}\right\rangle$ is smaller than the standard quantum limit (SQL) of $\frac{1}{4}$. Quantum-mechanical correlations between photons established through nonlinear interactions play an essential role in the generation of squeezed states of light.

Spin or angular momentum systems [2] have often been regarded as squeezed if the uncertainty of one spin component, say $\left\langle\Delta S_{x}{ }^{2}\right\rangle$ or $\left\langle\Delta S_{y}{ }^{2}\right\rangle$, is smaller than $\frac{1}{2}\left|\left\langle S_{z}\right\rangle\right|[3]$. This definition implies that a coherent spin state (CSS) [4] is already squeezed if it is placed in an appropriate system of coordinates, and also that spin can be squeezed by just rotating the CSS. Squeezed light emission from an atomic system in a certain CSS [5] has been regarded as evidence justifying this definition [3]. However, it is by no means obvious that we can judge the squeezing of spin by referring to the uncertainty of another (i.e., photon) system interacting with it. Moreover, the definition itself is problematic because it does not reflect quantum correlations but depends solely on the particular coordinate system. The reduced variance of a spin component does not necessarily mean squeezing in spin systems. The squeezing of spin is not as straightforward as the squeezing of bosons since their uncertainty relationships are essentially different [6].

The previous definition of squeezing in a spin system is also inappropriate from a practical viewpoint since it fails to correctly locate the SQL that is to be overcome by squeezing. It is known that an interferometer can be described as a spin system [7], yet the SQL of the interferometric phase sensitivity can never be overcome by a mere rotation of the CSS [8]. A spin state that improves the interferometric phase sensitivity beyond the SQL has been mathematically constructed $[7,8]$, and it is different from the CSS. These facts raise serious questions about whether CSS's are really qualified as squeezed states or whether there are other states that are more qualified to be called squeezed spin states (SSS's). Also, a prob- lem remains about how such states, if they exist, can be generated from experimentally available CSS's.

How to define and achieve spin squeezing is an important problem since spin can describe such diverse physical systems as the real spin of particles and magnons, collective two-level atoms [9], Cooper pairs in superconductors, and macroscopic two-state systems [10] like interferometers $[7,8]$, and Josephson junctions.

This paper establishes the concept of squeezed spin states (SSS's) and discusses the general principles for generating them. This paper is organized as follows. Section II describes the basic concept of spin squeezing in terms of quantum correlations among elementary spins. Section III proposes two mechanisms for spin squeezing and discusses their limits in quantum noise reduction. First, we propose a one-axis twisting mechanism as a building block for spin squeezing. Then, a two-axis countertwisting mechanism is introduced as a natural extension of one-axis twisting; this is shown to further reduce the quantum noise. Section IV discusses implementations of the twisting mechanisms in interferometers and two-level atoms. The Appendix derives some formulas that are necessary for calculating the moments.

\section{BASIC CONCEPT OF SQUEEZING IN SPIN SYSTEMS}

The spin or angular momentum system $\mathbf{S}=$ $\left(S_{x}, S_{y}, S_{z}\right)$ is governed by the cyclic commutation relations, $\left[S_{i}, S_{j}\right]=i \epsilon_{i j k} S_{k}$, where suffixes $i, j, k$ denote the components in any three orthogonal directions and $\epsilon_{i j k}$ is the Levi-Civita symbol. The associated uncertainty relationship is $\left\langle\Delta S_{i}{ }^{2}\right\rangle\left\langle\Delta S_{j}{ }^{2}\right\rangle \geq \frac{1}{4}\left|\left\langle S_{k}\right\rangle\right|^{2}$. A CSS $|\theta, \phi\rangle$ is defined as an eigenstate of a spin component in the $(\theta, \phi)$ direction, $S_{\theta, \phi}=S_{x} \sin \theta \cos \phi+S_{y} \sin \theta \sin \phi+S_{z} \cos \theta$, with eigenvalue $S$, where $\theta$ and $\phi$ denote the polar and azimuth angles. The CSS satisfies the minimum uncertainty relationship with uncertainties $\frac{S}{2}$ equally distributed over any two orthogonal components normal to the $(\theta, \phi)$ direction. Therefore, the spin vector $\mathbf{S}$ in a CSS can be conceived as a cone [11] as schematically shown in Fig. 1(a). In more rigorous words, the CSS has an isotropic quasiprobability distribution (QPD) [12] in a spherical phase space as shown in Figs. 2(a) and 3(a). 

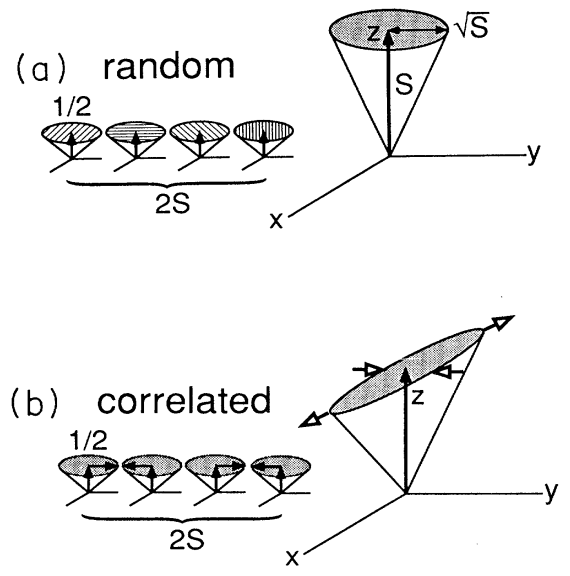

FIG. 1. Schematic illustrations of $S$-spin states in terms of $2 S$ individual $1 / 2$ spins. (a) Coherent spin state constructed from $2 S$ uncorrelated $1 / 2$ spins. (b) Squeezed spin state constructed from $2 S$ correlated $1 / 2$ spins.

An $S$-spin system can be regarded as a collective system consisting of $2 S$ elementary $\frac{1}{2}$ spins [13]. Any pure state of a $\frac{1}{2}$ spin is a CSS $\left|\theta_{k}, \phi_{k}\right\rangle_{k}=\cos \frac{\theta_{k}}{2}|\uparrow\rangle_{k}+$ $e^{i \phi_{k}} \sin \frac{\theta_{k}}{2}|\downarrow\rangle_{k}$, where $|\uparrow\rangle_{k}\left(|\downarrow\rangle_{k}\right)$ is the eigenstate of $S_{z}$ with the eigenvalue $\frac{1}{2}\left(-\frac{1}{2}\right)$ in the $k$ th $\frac{1}{2}$-spin system. The components normal to $\left(\theta_{k}, \phi_{k}\right)$ are completely uncertain, having a variance of $\frac{1}{4}$. The $S$-spin CSS $|\theta, \phi\rangle$ is equivalent to a set of $2 S$ elementary spins all pointing in the same mean direction $(\theta, \phi)$ as shown in Fig. 1(a). Since there are no quantum-mechanical correlations among these elementary spins, the variance of the components normal to the mean direction is simply the sum of the variances of the individual elementary spins, and is thus $\frac{S}{2}$. Now, if appropriate quantum-mechanical correlations are established among the elementary spins as schematically shown in Fig. 1(b), it is possible to partly cancel out fluctuations in one direction at the expense of those enhanced in the other direction. This is the basic concept of spin squeezing. The spin vector $\mathbf{S}$ in a squeezed spin state can be conceived as an elliptical cone [7] as schematically shown in Fig. 1(b). Such a state has an elliptical QPD in contrast to the isotropic one for a CSS. We regard spin as squeezed only if the variance of one spin component normal to the mean spin vector is smaller than the SQL of $\frac{S}{2}$. We have thus excluded mere mathematical coordinate dependency and included quantum correlation in our notion of squeezing. This notion naturally reflects the improved performance of spin systems.

To correlate the elementary spins requires a nonlinear interaction because a linear Hamiltonian merely rotates the individual spins and does not establish quantum correlations among them [14]. A $\frac{1}{2}$-spin system cannot be squeezed since it is equivalent to a system with only one elementary spin, which therefore has no partner to be correlated with.

\section{SPIN SQUEEZING BY NONLINEAR INTERACTIONS}

\section{A. One-axis twisting}

Now we demonstrate how the spin can be squeezed by nonlinear interaction. We consider a class of unitary transformations $U(t)=\exp \left[-i t F\left(S_{z}\right)\right]$ generated by the Hamiltonian $H=\hbar F\left(S_{z}\right)$ and see how they deform the noise distribution. The ladder operators $S_{ \pm} \equiv S_{x} \pm i S_{y}$ evolve as

$$
S_{+}(t)=U^{\dagger} S_{+}(0) U=S_{+}(0) \exp \left[i t f\left(S_{z}\right)\right]
$$

and $S_{-}(t)=\left[S_{+}(t)\right]^{\dagger}$, where

$$
f\left(S_{z}\right)=F\left(S_{z}+1\right)-F\left(S_{z}\right) .
$$

The lowest-order nonlinear interaction $F\left(S_{z}\right)=\chi S_{z}^{2}$ leads to $f\left(S_{z}\right)=2 \chi\left(S_{z}+\frac{1}{2}\right)$, rotation proportional to $S_{z}$, which twists the quantum fluctuations as shown in Fig. 2(b) [15]. This is analogous to self-phase modulation in the photon system [16]. The components after twisting are given by $\tilde{S}_{x}=\frac{1}{2}\left[S_{+} e^{i \mu\left(S_{z}+1 / 2\right)}+e^{-i \mu\left(S_{z}+1 / 2\right)} S_{-}\right]$and $\tilde{S}_{y}=\frac{1}{2 i}\left[S_{+} e^{i \mu\left(S_{z}+1 / 2\right)}-e^{-i \mu\left(S_{z}+1 / 2\right)} S_{-}\right]$, where $\tilde{S}_{i}$ and $S_{i}$ denote $S_{i}(t)$ and $S_{i}(0)$, and $\mu \equiv 2 \chi t$. With the CSS $\left|\frac{\pi}{2}, 0\right\rangle=2^{-S} \sum_{k=0}^{2 S}\left(\begin{array}{c}2 S \\ k\end{array}\right)^{1 / 2}|S, S-k\rangle$ as an initial state, we show in Figs. 2(b) and 2(c) how uncertainties are deformed by twisting as $\mu$ increases. Uncertainties are redistributed between certain orthogonal components in the $y-z$ plane.

Let us rotate the distribution around the $x$ axis by the unitary transformation $\overline{\mathbf{S}}=\exp \left(i \nu \tilde{S_{x}}\right) \tilde{\mathbf{S}} \exp \left(-i \nu \tilde{S_{x}}\right)$ to see how the uncertainties are redistributed. The means and variances become

$$
\begin{aligned}
\left\langle\bar{S}_{x}\right\rangle & =S \cos ^{2 S-1} \frac{\mu}{2}, \quad\left\langle\bar{S}_{y}\right\rangle=0, \quad\left\langle\bar{S}_{z}\right\rangle=0, \\
\left\langle\Delta \bar{S}_{x}^{2}\right\rangle & =\frac{S}{2}\left[2 S\left(1-\cos ^{2(2 S-1)} \frac{\mu}{2}\right)-\left(S-\frac{1}{2}\right) A\right],
\end{aligned}
$$

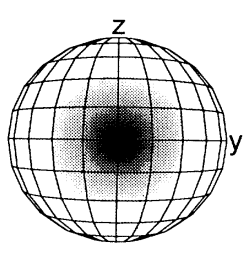

(a)

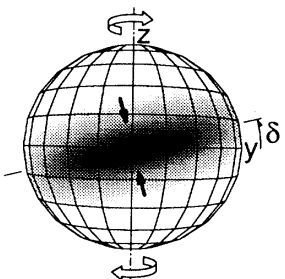

(b)

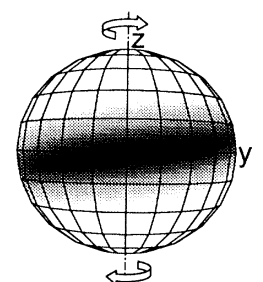

(c)
FIG. 2. State evolutions by one-axis twisting in terms of the quasiprobability distribution (QPD) on the sphere for $S=20$. The densities of the figures are normalized by the maximum value $Q_{\max }$ of $Q(\theta, \phi)$. (a) shows the initial coherent spin state $\left|\theta=\frac{\pi}{2}, \phi=0\right\rangle\left(Q_{\max }=1\right)$. (b) and (c) show one-axis twisted states generated by the unitary transformation $U=\exp \left[-i \mu S_{z}^{2} / 2\right]$; (b) optimally squeezed at $\mu=0.199$ $\left(Q_{\max }=0.445\right)$ and (c) excessively twisted at $\mu=0.399$ $\left(Q_{\max }=0.241\right)$. Although not clear from the figure, the QPD of (c) deviates from a geodesic (swirliness). 
$\left\langle\Delta \bar{S}_{z}^{2}\right\rangle=\frac{S}{2}\left\{1+\frac{1}{2}\left(S-\frac{1}{2}\right)\left[A \pm \sqrt{A^{2}+B^{2}} \cos (2 \nu+2 \delta)\right]\right\}$,

where we define $A=1-\cos ^{2 S-2} \mu, B=4 \sin \frac{\mu}{2} \cos ^{2 S-2} \frac{\mu}{2}$, and $\delta=\frac{1}{2} \arctan \frac{B}{A}$. Equation (3) shows the anisotropically distributed quantum fluctuations of the SSS. Our primary concern is to minimize one of the variances in $\mathrm{Eq}$. (3). The term $\left\langle\Delta \bar{S}_{y}^{2}\right\rangle$ is minimized and $\left\langle\Delta \bar{S}_{z}^{2}\right\rangle$ is maximized when $\nu=\frac{\pi}{2}-\delta$. Likewise $\left\langle\Delta \bar{S}_{z}^{2}\right\rangle$ is minimized and $\left\langle\Delta \bar{S}_{y}^{2}\right\rangle$ is maximized when $\nu=-\delta$. The increased (upper sign) and decreased (lower sign) variances are written as

$\left.V_{ \pm}=\frac{S}{2}\left\{\left[1+\frac{1}{2}\left(S-\frac{1}{2}\right) A\right] \pm \frac{1}{2}\left(S-\frac{1}{2}\right) \sqrt{A^{2}+B^{2}}\right]\right\}$.

For $S \gg 1$ and $|\mu| \ll 1$, they can be approximated as

$$
V_{+} \approx \frac{S}{2} 4 \alpha^{2}, \quad V_{-} \approx \frac{S}{2}\left(\frac{1}{4 \alpha^{2}}+\frac{2}{3} \beta^{2}\right)
$$

where we have set $\alpha=\frac{1}{2} S \mu$ and $\beta=\frac{1}{4} S \mu^{2}$ and assumed $|\alpha|>1$ and $\beta \ll 1$, but kept the terms up to the lowest order in $\beta$ to take into account the spherical nature of the phase space and the swirliness of the QPD - the deviations from a geodesic. Also we get $\delta \approx \frac{1}{2} \arctan (1 / \alpha)$, $\left\langle\overline{S_{x}}\right\rangle \approx S(1-\beta)$, and $\left\langle\Delta{\overline{S_{x}}}^{2}\right\rangle \approx 2 \alpha^{2}$. The uncertainty $\sqrt{V_{-}}$is suppressed by a factor of $2|\alpha|$ compared to that of the initial CSS, while $\sqrt{V_{+}}$is enhanced by the same factor. The reduced variance $V_{-}$reaches its minimum $V_{\min } \approx \frac{1}{2}\left(\frac{S}{3}\right)^{1 / 3}$ at $|\mu|=\mu_{0}=24^{1 / 6} S^{-2 / 3}$ when the second term on the right-hand side of Eq. (5) becomes comparable to the first term. The exact minimum attainable variances obtained from Eq. (4) are plotted as a function of $S$ in Fig. 4. They are even smaller than the above approximate expression for small $S$ and asymptotically approach it for large $S$. The normalized uncertainty product $U_{y z} \equiv 4\left\langle\Delta S_{y}{ }^{2}\right\rangle\left\langle\Delta S_{z}{ }^{2}\right\rangle /\left|\left\langle S_{x}\right\rangle\right|^{2}$ is calculated to be $U_{y z} \approx 1+\left(\frac{\mu}{\mu_{0}}\right)^{6}$; therefore, the state remains almost in the minimum uncertainty state for $|\mu|<\mu_{0}$. Figure 2(b) shows the QPD of the optimally squeezed state which gives the minimum variance for $S=20$ and Fig. 2(c) shows that of an excessively twisted state. The minimum attainable uncertainty of one-axis twisting is limited by the swirliness of the QPD.

\section{B. Two-axis countertwisting}

The swirliness cancels out if the QPD is simultaneously twisted clockwise and counterclockwise about two orthogonal axes, both normal to the mean spin vector of the initial CSS as shown in Fig. 3(b). Let us squeeze the initial CSS $|0, \phi\rangle$ with respect to $S_{x}$ and $S_{y}$ by twisting it about the two axes in the $\theta=\frac{\pi}{2}, \phi= \pm \frac{\pi}{4}$ directions. The Hamiltonian of two-axis countertwisting is written as

$$
H=\hbar \chi\left(S_{\frac{\pi}{2}, \frac{\pi}{4}}^{2}-S_{\frac{\pi}{2},-\frac{\pi}{4}}^{2}\right)=\frac{\hbar \chi}{2 i}\left(S_{+}^{2}-S_{-}^{2}\right) .
$$

The minimum attainable variance of the two-axis SSS is less than $\frac{1}{2}$ for small $S$ and asymptotically approaches $\frac{1}{2}$

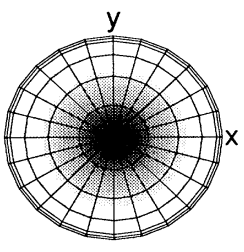

(a)

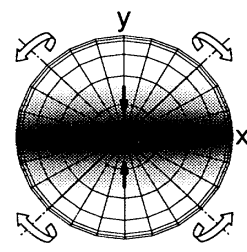

(b)

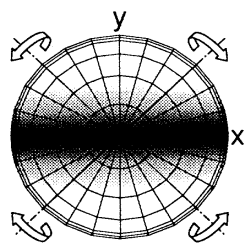

(c)
FIG. 3. State evolutions by two-axis countertwisting in terms of the quasiprobability distribution (QPD) on the sphere for $S=20$. The densities of the figures are normalized by the maximum value $Q_{\max }$ of $Q(\theta, \phi)$. (a) shows the initial coherent spin state $|\theta=0, \phi\rangle\left(Q_{\max }=1\right)$. (b) and (c) are two-axis countertwisted states generated by the unitary transformation $U=\exp \left[-i \mu\left(S_{\frac{\pi}{2}, \frac{\pi}{4}}^{2}-S_{\frac{\pi}{2},-\frac{\pi}{4}}^{2}\right) / 4\right]$; (b) optimally squeezed at $\mu=0.203\left(Q_{\max }=0.252\right)$ and (c) excessively twisted at $\mu=0.248\left(Q_{\max }=0.187\right)$, where the QPD splits into two parts.

as $S$ increases, as shown in Fig. 4. The QPD shown in Fig. 3(b) is that of the optimally squeezed state that gives the minimum variance for $S=20$. The QPD is shrunk along a geodesic on the sphere and stretched along the orthogonal geodesic. When the QPD spans almost half of the sphere, the reduced variance attains a minimum of $\frac{1}{2}$ while the enhanced one reaches $\frac{S^{2}}{2}$. As the length of the mean spin vector remains on the order of $S$, the state is still close to the minimum uncertainty state. If $\mu=4 \chi t$ exceeds the optimal value, the QPD splits into two parts as shown in Fig. 3(c).

In spin systems, the squeezing occurs on the phase sphere (spherical phase space). Unlike boson squeezing, the QPD cannot be homogeneously or globally squeezed in one direction over the whole phase space. If a spin

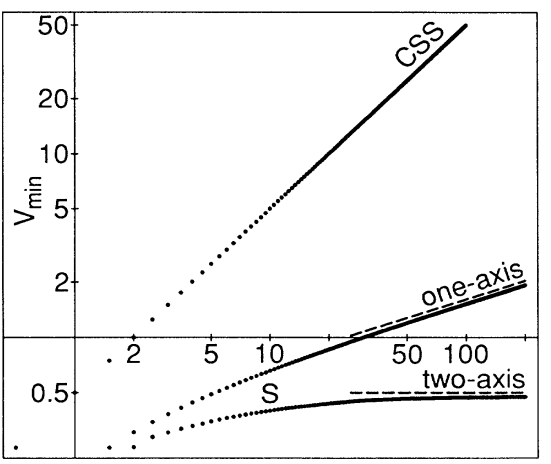

FIG. 4. The minimum attainable variances of the spin component normal to the mean spin vector as a function of $S$ for one-axis squeezed spin states and two-axis squeezed spin states. They asymptotically approach $\frac{1}{2}\left(\frac{S}{3}\right)^{1 / 3}$ and $\frac{1}{2}$ (broken lines) for increasing $S$. The special case of $S=1$ where they reach 0 is not shown. The variances of coherent spin states, $\frac{S}{2}$, are also plotted for comparison. 
component is shrunk around a certain point on the sphere, it must be stretched around another point. This imposes a fundamental restriction on the reduction in quantum noise. The two-axis countertwisting mechanism is the one that achieves the maximal noise reduction. The $S=1$ spin system is an exception where both one-axis and two-axis twisting can completely squeeze out the uncertainty of one component and generate the eigenstate of that component with the eigenvalue 0 .

\section{IMPLEMENTATIONS}

Finally, we briefly discuss implementations of our twisting Hamiltonians in two-state systems.

\section{A. Interferometers}

Let us consider $N$ quanta partitioned by a $50 \%$ beamsplitter into two outputs, $A$ and $B$. Each quantum is in a superposition of the state in which the particle exists in $A$ but not in $B$ and the state in which it exists in $B$ but not in $A$, as shown in Fig. 5 . The former (latter) is an eigenstate of $\sigma_{k}^{z}=\frac{1}{2}\left(a_{k}^{\dagger} a_{k}-b_{k}^{\dagger} b_{k}\right)$ with eigenvalue $\pm \frac{1}{2}$ which corresponds to $|\uparrow\rangle(|\downarrow\rangle)$ of $\frac{1}{2}$ spin. Beamsplitters and phase shifters rotate this abstract "spin."

At the input port of the beamsplitter, $N$ particles all exist in $A$, namely, all $N$ of the $\frac{1}{2}$ spins are in $|\uparrow\rangle$. This is a $\operatorname{CSS}|\theta=0, \phi\rangle$ of $\frac{N}{2}$ spin. It is also an eigenstate of $\mathrm{S}^{2}$ with eigenvalue $\frac{N}{2}\left(\frac{N}{2}+1\right)$ and continues to be so as long as the Hamiltonian commutes with $\mathbf{S}^{2}$. For example, dispersion-less beamsplitters and phase shifters rotate all $N$ spins in the same manner and preserve $\mathbf{S}^{2}$. Under these conditions, the system continues to behave as a spin $\frac{N}{2}$.

The mathematical relationships between the partitioned $N$ quanta and spin $\frac{N}{2}$ are $[7,8]$

$S_{+} \equiv \sum_{k} a_{k}^{\dagger} b_{k}=S_{x}+i S_{y}$

$S_{z} \equiv \frac{1}{2}\left(N_{A}-N_{B}\right) \quad\left(N_{A} \equiv \sum_{k} a_{k}^{\dagger} a_{k}, N_{B} \equiv \sum_{k} b_{k}^{\dagger} b_{k}\right)$.

The creation and annihilation operators $a_{k}^{\dagger}$ and $a_{k}$ express the quasimonochromatic field of arm $A$, and $b_{k}^{\dagger}$ and $b_{k}$ those of $B$, which obey either boson commutation re-

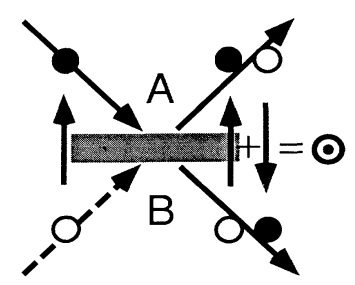

FIG. 5. A particle in an interferometer as an abstract spin $1 / 2$. Filled circles mean that a particle exists and open circles mean that it does not. The arrow indicating which arm the particle exists in behaves like a spin $1 / 2$. lations or fermion anticommutation relations. The operator $S_{+}$coherently (without changing mode index $k$ ) transfers a particle from $B$ to $A$ and its phase represents the relative phase of $A$ and $B$. The vertical component $S_{z}$ represents half of the particle number difference between $A$ and $B$.

How can we twist this spin $\frac{N}{2}$ system? Physically, we can twist the spin about the $z$ axis by modulating the relative phase of $A$ and $B$ by the population difference between $A$ and $B$.

For example, if the phase of $A$ varies in proportion to $N_{A}$ and that of $B$ in proportion to $N_{B}$, then the relative phase is modulated by the population difference. For photons, this can be achieved by inserting an optical Kerr medium into each arm and turning on the self-phase modulations [16]. The interaction Hamiltonian is given by $H_{I}=\hbar \chi\left(N_{A}^{2}+N_{B}^{2}\right)=2 \hbar \chi\left(N^{2} / 4+S_{z}^{2}\right)$, which performs one-axis twisting since $N$ is a constant of motion.

Alternatively, if the phase of $A$ varies in proportion to $N_{B}$ and that of $B$ in proportion to $N_{A}$, the result is also modulation of the relative phase by the population difference, but the sign is opposite. For charged particles, this can be achieved as a mutual phase modulation due to Coulomb interaction between particles of each arm [17]. The interaction Hamiltonian is given by $H_{I}=\hbar \chi N_{A} N_{B}=\hbar \chi\left(N^{2} / 4-S_{z}^{2}\right)$, which performs oneaxis twisting.

The two-axis countertwisting Hamiltonian in Eq. (6) suggests an interaction that coherently transfers two particles at the same time. This might be achieved by fourwave mixing [7].

What is the merit of squeezing this spin $\frac{N}{2}$ system? When $N$ quanta are equally partitioned, i.e., $\left\langle S_{z}\right\rangle=0$, the uncertainties of particle-number difference and phase difference are given by

$$
\begin{aligned}
\delta N & \equiv\left\langle\left[\Delta\left(N_{A}-N_{B}\right)\right]^{2}\right\rangle=2\left\langle\Delta S_{z}^{2}\right\rangle^{1 / 2}, \\
\delta \phi & \equiv\left\langle\left[\Delta\left(\phi_{A}-\phi_{B}\right)\right]^{2}\right\rangle \approx\left\langle\Delta S_{y}^{2}\right\rangle^{1 / 2} /\left|\left\langle S_{x}\right\rangle\right|,
\end{aligned}
$$

assuming that the mean spin vector $\langle\mathbf{S}\rangle$ is parallel to the $x$ axis [17]. The uncertainty relationship $\delta N \delta \phi \geq 1$ follows from the spin uncertainty relationships. A phase difference smaller than $\delta \phi$ is not detectable by interferometric measurement. For an ordinary $50 \%$ linear beamsplitter, $\delta N=\sqrt{N}$ and $\delta \phi \approx 1 / \sqrt{N}$ because the outputs form an CSS $\left|\frac{\pi}{2}, 0\right\rangle$. Spin squeezing can reduce $\delta \phi$ without violating the uncertainty relationship and therefore it improves the interferometric sensitivity.

This is a totally new possibility for fermions since no fermion analog of boson squeezed state [18] has been found [19]. The application of spin squeezing in partition and interferometry will be discussed in detail elsewhere [20].

\section{B. Two-level atoms}

A collection of $N$ two-level atoms can be described as a spin system of $S=\frac{N}{2}$ [9]. Correspondence is basically the same as above by replacing the $\operatorname{arm} A$ with the upper state $|A\rangle, B$ with the lower state $|B\rangle$, and the $k$ th mode with the $k$ th atom. Each atom is represented by an ab- 
stract spin $\frac{1}{2}$. The populations of the upper and lower states are $N_{A}$ and $N_{B}$, respectively. The vertical component $S_{z}$ corresponds to half the population difference. The horizontal components $S_{x}$ and $S_{y}$ represent the two quadrature-phase amplitudes of the dipole moment.

If all of the atoms are in the upper (lower) state, the collective system is in a CSS $|\theta=0, \phi\rangle(|\theta=\pi, \phi\rangle)$. The dipole interaction of the collective atomic system with classical electromagnetic wave rotates the spin vector [4], but does not offer spin squeezing.

The one-axis twisting Hamiltonian corresponds to the energy propotional to the square of the population difference. The two-axis twisting Hamiltonian corresponds to the simultaneous excitation-deexcitation of two atoms. Although realistic physical schemes are yet to be found, these nonlinear Hamiltonians will provide some clues in the search for squeezed atomic states [21].

\section{CONCLUSIONS}

In conclusion, we have clarified the concept of squeezing in spin systems. An $S$-spin system is squeezed only if one of the components normal to the mean spin vector has a variance smaller than $S / 2$. We have shown the principle for spin squeezing. The spin can be squeezed by establishing quantum correlations among elementary spins. We have proposed two fundamental mechanisms for spin squeezing, and discussed their limits of noise re- duction. One-axis twisting can reduce the noise down to the order of $S^{1 / 3}$ and two-axis countertwisting can reduce it to $1 / 2$. We have also discussed implementations of spin squeezing in interferometers.

\section{APPENDIX: CALCULATIONS OF TWISTED MOMENT}

In this appendix, we derive some formulas for calculating the moments of twisted spin operators. A coherent spin state $|\theta, \phi\rangle$ can be expanded in terms of $S_{z}$ eigenstates $|S, k\rangle$ as follows [4]:

$$
\begin{aligned}
|\theta, \phi\rangle= & \left(1+\tan ^{2} \frac{\theta}{2}\right)^{-S} \\
& \times \sum_{k=0}^{2 S}\left(e^{i \phi} \tan \frac{\theta}{2}\right)^{k}\left(\begin{array}{c}
2 S \\
k
\end{array}\right)^{1 / 2}|S, S-k\rangle .
\end{aligned}
$$

In the following, we assume that the initial state is a CSS:

$$
\left|\frac{\pi}{2}, 0\right\rangle=2^{-S} \sum_{k=0}^{2 S}\left(\begin{array}{c}
2 S \\
k
\end{array}\right)^{1 / 2}|S, S-k\rangle,
$$

and write the expectation value of an operator $O$ with respect to this state simply as $\langle O\rangle$.

The first moments of the spin components are calculated from

$$
\begin{aligned}
\left\langle S_{+} \exp \left[i \mu\left(S_{z}+\frac{1}{2}\right)\right]\right\rangle & =2^{-2 S} \sum_{k=0}^{2 S} \sum_{l=0}^{2 S}\left(\begin{array}{c}
2 S \\
k
\end{array}\right)^{1 / 2}\left(\begin{array}{c}
2 S \\
l
\end{array}\right)^{1 / 2}\left\langle S, S-k\left|S_{+} \exp \left[i \mu\left(S_{z}+\frac{1}{2}\right)\right]\right| S, S-l\right\rangle \\
& =S\left(\cos \frac{\mu}{2}\right)^{2 S-1}
\end{aligned}
$$

and its complex conjugate.

The calculation of the second moments requires the following two formulas. In a similar manner as Eq. (A1), we obtain

$\left\langle\left\{S_{+} \exp \left[i \mu\left(S_{z}+\frac{1}{2}\right)\right]\right\}^{2}\right\rangle=S\left(S-\frac{1}{2}\right)(\cos \mu)^{2 S-2}$.

Differentiating Eq. (A1) with respect to $\mu$ yields

$$
\begin{aligned}
\left\langle i S_{+} \exp \left[i \mu\left(S_{z}+\frac{1}{2}\right)\right]\left(S_{z}+\frac{1}{2}\right)\right\rangle & \\
& =-S\left(S-\frac{1}{2}\right)\left(\cos \frac{\mu}{2}\right)^{2 S-2} \sin \frac{\mu}{2} .
\end{aligned}
$$

[1] For special issues on squeezed light, see J. Opt. Soc. Am. B 4, 1450 (1987), edited by H. J. Kimble and D. F. Walls; R. Loudon and P. L. Knight, J. Mod. Opt. 34 (1987).

[2] M. E. Rose, Elementary Theory of Angular Momentum (Wiley, New York, 1957).

[3] K. Wódkiewicz and J. H. Eberly, J. Opt. Soc. Am. B 2, 458 (1987); K. Wódkiewicz, Phys. Rev. B 32, 4750 (1985).

[4] J. M. Radcliffe, J. Phys. A 4, 313 (1971); F. T. Arecchi, E. Courtens, R. Gilmore, and H. Thomas, Phys. Rev. A
6, 2211 (1972). For review, see J. R. Klauder and B. S. Skagerstam, Coherent States (World Scientific, Singapore, 1985).

[5] D. F. Walls and P. Zoller, Phys. Rev. Lett. 47, 709 (1981).

[6] C. Aragone, E. Chalbaud, and S. Salamó, J. Math. Phys. 17, 1963 (1976).

[7] B. Yurke, S. L. McCall, and J. R. Klauder, Phys. Rev. A 33, 4033 (1986).

[8] B. Yurke, Phys. Rev. Lett. 56, 1515 (1986); Physica 
151B, 286 (1988).

[9] R. H. Dicke, Phys. Rev. 93, 99 (1954).

[10] R. P. Feynman, R. B. Leighton, and M. Sands, The Feynman Lectures on Physics, Vol. III (Quantum Mechanics) (Addison-Wesley, Reading, MA, 1965).

[11] D. Bohm, Quantum Theory (Prentice-Hall, New York, 1951), p. 318.

[12] The quasiprobability distribution (QPD) for state $|\Psi\rangle$ is defined as $Q(\theta, \phi)=|\langle\theta, \phi \mid \Psi\rangle|^{2}$.

[13] L. D. Landau and E. M. Lifshitz, Quantum Mechanics (Pergamon, London, 1958).

[14] Quantum correlations can be also established by interaction with already correlated systems such as squeezed light. However, this paper focuses on the squeezing of a spin system without relying on the other correlated systems.

[15] Higher-order nonlinearities also give rise to a certain kind of squeezing. If $f\left(S_{z}\right)$ is an odd function of $S_{z}$, it causes twisting basically similar to that discussed in the present paper. Even functions other than a constant cause twist- ing that is symmetrical about the equator.

[16] M. Kitagawa and Y. Yamamoto, Phys. Rev. A 34, 3974 (1986).

[17] M. Kitagawa and M. Ueda, Phys. Rev. Lett. 67, 1852 (1991).

[18] C. M. Caves, Phys. Rev. D 23, 1693 (1981).

[19] Squeezed fermion states defined by K. Svozil, Phys. Rev. Lett. 65, 3341 (1990), cannot improve the interferometric phase sensitivity because of the Pauli exclusion principle.

[20] M. Kitagawa and M. Ueda (unpublished). Nonlinear interferometers based on spin squeezing have been partly presented in International Conference on Quantum Electronics, Vienna, 1992 [Tech. Dig. Ser. 9, 26 (1992)], by the same authors.

[21] The multiatom squeezed states discussed in the following papers are quite different from the SSS except for the special case of $S=1$ : S. M. Barnett and M.-A. Dupertuis, J. Opt. Soc. Am. B 2, 505 (1987); M.-A. Dupertuis, S. M. Barnett, and S. Stenholm, ibid. 4, 1124 (1987). 